\title{
A Double-Pulsar System - A Rare Laboratory for Relativistic Gravity and Plasma Physics
}

\author{
A.G. Lyne, ${ }^{1 *}$ M. Burgay, ${ }^{2}$ M. Kramer, ${ }^{1}$ A. Possenti, ${ }^{3,4}$ \\ R.N. Manchester, ${ }^{5}$ F. Camilo, ${ }^{6}$ M.A. McLaughlin, ${ }^{1}$ D.R. Lorimer, ${ }^{1}$ \\ N. D'Amico, ${ }^{3,7}$ B.C. Joshi, ${ }^{8}$ J. Reynolds ${ }^{9}$ and P.C.C. Freire ${ }^{10}$ \\ ${ }^{1}$ University of Manchester, Jodrell Bank Observatory, Macclesfield, SK11 9DL, UK \\ ${ }^{2}$ Università degli Studi di Bologna, Dipartimento di Astronomia, via Ranzani 1, \\ 40127 Bologna, Italy \\ ${ }^{3}$ INAF - Osservatorio Astronomico di Cagliari, Loc. Poggio dei Pini, Strada 54, \\ 09012 Capoterra, Italy \\ ${ }^{4}$ INAF - Osservatorio Astronomico di Bologna, via Ranzani 1, 40127 Bologna, Italy \\ ${ }^{5}$ Australia Telescope National Facility, CSIRO, P.O. Box 76, Epping, NSW 1710, Australia \\ ${ }^{6}$ Columbia Astrophysics Laboratory, Columbia University, 550 West 120th Street, \\ New York, NY 10027, USA \\ ${ }^{7}$ Università degli Studi di Cagliari, Dipartimento di Fisica, SP Monserrato-Sestu km 0.7, \\ 09042 Monserrato, Italy \\ ${ }^{8}$ National Centre for Astrophysics, P.O. Bag 3, Ganeshkhind, Pune 411007, India \\ ${ }^{9}$ ATNF, Parkes Observatory, P.O. Box 276, Parkes, NSW 2870, Australia \\ ${ }^{10}$ NAIC, Arecibo Observatory, HC03 Box 53995, PR 00612, USA
}

*To whom correspondence should be addressed; E-mail: agl@jb.man.ac.uk

The clock-like properties of pulsars moving in the gravitational fields of their unseen neutron-star companions have allowed unique tests of general relativity and provided evidence for gravitational radiation. We report here the detection of the 2.8-sec pulsar J0737-3039B as the companion to the 23-ms 
pulsar J0737-3039A in a highly-relativistic double-neutron-star system, allowing unprecedented tests of fundamental gravitational physics. We observe a short eclipse of J0737-3039A by J0737-3039B and orbital modulation of the flux density and pulse shape of J0737-3039B, probably due to the influence of J0737-3039A's energy flux upon its magnetosphere. These effects will allow us to probe magneto-ionic properties of a pulsar magnetosphere.

Double-neutron-star (DNS) binaries are rare, and only six such systems are known. However, they can provide wonderful laboratories for the study of relativistic gravity and gravitational radiation (11). The discovery of such systems has been a prime objective of pulsar surveys since the first, PSR B1913+16, was discovered 29 years ago (2). Apart from their rarity, they are particularly difficult to find because of the large orbital acceleration experienced by the members of the systems, resulting in large and varying Doppler effects in the observed rotational period.

The pulsar J0737-3039 was recently discovered as part of a high-latitude multibeam survey of the southern sky using the 64-m Parkes radio telescope (3). It was found to be in a 2.4-hr eccentric orbit with another compact object that the observed orbital parameters suggested was another neutron star. The short orbital period and compactness of the system and the high timing precision made possible by the large flux density and narrow pulse features of this pulsar promise to make this system a superb laboratory for the investigation of relativistic astrophysics. Relativistic advance of the angle of periastron has already been measured and orbital decay due to gravitational wave emission will be measured with high precision within a few months. The resulting in-spiral will end in coalescence of the two stars in about $85 \mathrm{My}(3)$. This discovery significantly increases the estimates of the detection rate of DNS in-spirals by gravitational wave detectors $(3,4)$. 
The discovery of PSR J0737-3039B. No other pulsar was detected in the 4.5-min discovery observation of PSR J0737-3039. However, analysis of data acquired subsequently for detailed studies of the system has revealed the occasional presence of pulsations with a period of 2.8 seconds. This pulsar, henceforth called PSR J0737-3039B (or simply B in this paper), has the same dispersion measure (the integrated column density of free electrons along the line of sight) as the original pulsar, henceforth called PSR J0737-3039A (or simply A) and shows Doppler variations in the pulse period which identify it as the companion.

The data obtained by Burgay et al. (3) for their studies of A used the Parkes radio telescope at a frequency of $1390 \mathrm{MHz}$ and have now all been reprocessed to enable investigation of the properties of B. Additionally, the system was observed on seven days in November 2003 simultaneously with dual-polarization receivers centered on frequencies of $680 \mathrm{MHz}$ and $3030 \mathrm{MHz}$ (5). Observations were also made of both pulsars at $1396 \mathrm{MHz}$ with the 76-m Lovell telescope at Jodrell Bank, also with a dual-polarization receiver. Timing observations of both pulsars have been made covering the 7 months from May to November 2003, with individual data spans between 10 minutes and 5 hours.

The main features of the observations of B are shown in Fig. 1, which illustrates the strength of the 2.8-sec pulsed emission in the three frequency bands as a function of orbital phase and pulse phase over the full orbit. Most notable is the variation in the received flux density of the pulsar, that is clearly visible for two brief periods of about 10 minutes duration each centered upon orbital longitudes $210^{\circ}$ and $280^{\circ}$. Within these bursts, the strength is such that most pulses are detected individually. In addition to the orbital phases where it is always detected, the pulsar often shows weak emission elsewhere, notably between orbital phases $0^{\circ}$ and $20^{\circ}$ (Fig. 1B). It is likely that observations with greater sensitivity will reveal more widespread emission throughout the orbit. The pattern of the visibility is essentially stable from orbit to orbit and from band to band over the full frequency range of $680 \mathrm{MHz}$ to $3030 \mathrm{MHz}$. The absence 
of pulsar B in the discovery observation of A can now be understood, as that observation was made at a longitude of $146^{\circ}$, where there is little emission from B.

There are significant changes in the shape of the pulses of B with orbital phase (Fig. 1). The data at $1390 \mathrm{MHz}$ show that it varies from a narrow intense main pulse with a weak precursor in the burst around longitude $210^{\circ}$, to a roughly-equal double-component profile with a somewhat greater separation near longitude $280^{\circ}$. It becomes essentially a single pulse at around longitude $0^{\circ}$. There is also an indication for frequency evolution of pulse shape within the bursts. The double nature of the profiles becomes more evident at the higher frequency of $3030 \mathrm{MHz}$ and less so at $680 \mathrm{MHz}$, where the components seem to have nearly merged.

In order to make timing measurements of the pulsar, a phase-dependent set of pulse templates was created by forming average profiles for each of the three burst regions. These templates were aligned in such a way that the trailing, usually more intense, components were at the same pulse phase. Cross-correlation of data with appropriate templates from this set was carried out to produce times of arrival (TOAs). These TOAs were then compared with a model for the astrometric, rotational and orbital parameters using the TEMPO pulsar timing program (6). The position and main orbital parameters have been determined from the high-precision timing enabled by the strong, narrow pulses of A (Table 1 ). For B, an offset of $180^{\circ}$ was added to the longitude of periastron and the only fitted parameters were the pulsar rotational period, $P$, its first derivative, $\dot{P}$, and the projected semi-major axis, $x_{\mathrm{B}}=a_{\mathrm{B}} \sin i / c$, where $i$ is the orbital inclination and $c$ is the speed of light (Table 1).

Gravitational Physics. With their strong gravitational fields and rapid motions, DNS binaries exhibit large relativistic effects. General relativity and other theories of gravity can be tested when a number of relativistic corrections, the so-called post-Keplerian (hereafter PK) parameters, to the classical Keplerian description can be measured. In this formalism, for point masses 
with negligible spin contributions, the PK parameters in each theory should only be functions of the a priori unknown neutron star masses and the classical Keplerian parameters. With the two masses as the only free parameters, the measurement of three or more PK parameters overconstrains the system, and thereby provides a test-ground for theories of gravity (7). In a theory that describes the binary system correctly, the PK parameters define lines in a mass-mass diagram that all intersect in a single point. Such tests have been possible to date in only two DNS systems, PSR B1913+16 (8) and PSR B1534+12 (9) (see also (10)). For PSR B1913+16, the relativistic periastron advance, $\dot{\omega}$, the orbital decay due to gravitational wave damping, $\dot{P}_{\mathrm{b}}$, and the gravitational redshift/time dilation parameter, $\gamma$, have been measured, providing a total of three PK parameters. For PSR B1534+12, Shapiro delay (11), caused by passage of the pulses through the gravitational potential of the companion, is also visible, since the orbit is seen nearly edge-on. This results in two further PK parameters, $r$ ("range") and $s$ ("shape") of the Shapiro delay. However, the observed value of $\dot{P}_{\mathrm{b}}$ requires correction for kinematic effects (12), so that PSR B1534+12 provides four PK parameters usable for precise tests (9).

Extending and improving the timing solution for A (3), we have measured A's $\dot{\omega}$ and $\gamma$ and have also detected the Shapiro delay in the pulse arrival times of A due to the gravitational field of B (Fig. 2). This provides four measured PK parameters, resulting in a $m_{A}-m_{B}$ plot (Fig. 3) through which we can test the predictions of general relativity (13, 11). However, the detection of $\mathrm{B}$ as a pulsar opens up opportunities to go beyond what is possible with previously known DNS binary systems. Firstly, we can exclude all regions in the $m_{\mathrm{A}}-m_{\mathrm{B}}$ plane that are forbidden by the individual mass functions of $\mathrm{A}$ and $\mathrm{B}$ due to the requirement $\sin i \leq 1$. Secondly, with a measurement of the projected semi-major axes of the orbits of A and B (Table 1), we obtain a precise measurement of the mass ratio, $R\left(m_{\mathrm{A}}, m_{\mathrm{B}}\right) \equiv m_{\mathrm{A}} / m_{\mathrm{B}}=x_{\mathrm{B}} / x_{\mathrm{A}}$, providing a further constraint in the $m_{A}-m_{B}$ plot (Fig. 3). This relation is valid for any theory of gravity (7 14). Most importantly, the $R$-line is independent of strong-field (self-field) effects, 
providing a stringent and new constraint for tests of gravitational theories (13 11 15).

With four PK parameters already available for tests, this additional constraint makes this system the most over-determined DNS binary to date and a truly unique laboratory for relativistic gravity. Moreover, with a significant measurement of $\dot{P}_{\mathrm{b}}$ expected within the next few months, an additional PK parameter will become available. This may provide a sixth constraint if kinematic effects are negligible or can be isolated by proper motion and distance measurements. The position of the allowed region in Fig. 3 also determines the inclination of the orbit to the line-of-sight. It turns out that the system is observed nearly edge-on with an inclination angle $i$ of about $87^{\circ}$ (Table 1 ).

Due to the curvature of space-time near massive objects, the spin axes of both pulsars will precess about the total angular momentum vector, changing the orientation of the pulsars as seen from Earth (16). With the measured system parameters (Table 1), general relativity predicts periods of such geodetic precession of only $75 \mathrm{yr}$ for A and $71 \mathrm{yr}$ for B (17). Hence, the relative orientation of the pulsars' spin axes within the system geometry is expected to change on short time-scales. This should lead to measurable changes in the profiles of A and B (cf. (18 |19|20)), and perhaps also to measurable changes in the aberration effects due to the rotation of $\mathrm{A}$ and $\mathrm{B}$. Hence, we can expect that additional PK parameters can be measured that are too small to be detected in other binary pulsars. One example of these are the aberration terms $A, B$ and $\delta_{r}$ of the DD timing model that we used (13 11), assumed to be zero for the present analysis.

In contrast to previous tests of general relativity, we may soon have to use higher order terms than $(v / c)^{2}$ to describe the system accurately, the first time this has been necessary for binary pulsar systems. The future high precision of the measurement of $\dot{\omega}$ may demand this first for comparison of the observed value of this parameter with theories of gravity (21). Deviations from the value predicted by general relativity may be caused by contributions from spin-orbit coupling (17), which is about an order of magnitude larger than for PSR B1913+16. This 
potentially will allow us to measure the moment of inertia of a neutron star for the first time (21. 22).

Origin and evolution of the double-pulsar system. The existence of DNS binaries can be understood by a binary evolution scenario which starts with two main sequence stars (e.g. (23)). The initially more massive star evolves first and eventually explodes in a supernova to form a neutron star. Under favorable conditions, this neutron star remains bound to its companion and spins down as a normal pulsar for the next $10^{6}-10^{7} \mathrm{yr}$. At some later time, the remaining (secondary) star comes to the end of its main-sequence lifetime and begins a red giant phase. Depending on the orbital parameters of the system, the strong gravitational field of the neutron star attracts matter from the red giant, forming an accretion disk and making the system visible as an X-ray binary. The accretion of matter transfers orbital angular momentum to the neutron star, spinning it up to short periods and dramatically reducing its magnetic field (24 25). A limiting spin period is reached due to equilibrium between the magnetic pressure of the accreting neutron star and the ram pressure of the infalling matter (23. 26).

A crucial phase in the evolution of close DNS binaries like J0737-3039 is the dramatic reduction in orbital separation that occurs when matter from the secondary star is expelled from the system, resulting in a very compact system consisting of a helium star and a neutron star (27 28). A sufficiently massive helium star will ultimately undergo a supernova explosion forming a young, second neutron star. If the stars remain bound following this explosion, the resulting system is a pair of neutron stars in an eccentric orbit with very different magnetic field strengths and hence spin-down properties, as in fact observed here. With masses of $m_{\mathrm{A}}=$ $1.34 \mathrm{M}_{\odot}$ and $m_{\mathrm{B}}=1.25 \mathrm{M}_{\odot}, \mathrm{A}$ is typical of other neutron stars with measured masses (29, 10), while B has a significantly smaller mass than any other.

The time since the second supernova explosion can be estimated by comparing our mea- 
surements of $P$ and $\dot{P}$ for $\mathrm{A}$ and $\mathrm{B}$, which can be used to compute their characteristic ages $\tau=P /(2 \dot{P})$. If characteristic ages are good indicators of pulsars' true ages, we expect $\tau_{\mathrm{A}}=\tau_{\mathrm{B}}$, but the observed values are $\tau_{\mathrm{A}} \simeq 4 \tau_{\mathrm{B}}$. This discrepancy can be reconciled by questioning one or more of the assumptions inherent in the use of characteristic ages as estimates of true ages: a negligible birth spin period and a non-decaying magnetic dipole braking torque. At the very least, the post-accretion spin period of A cannot have been negligible due to details of the accretion process discussed above. Simple models assuming constant magnetic dipole spindown (26) predict a post-accretion spin period for A in the range 10-18 ms, enough to explain the observed discrepancy in the characteristic ages.

Probing pulsar magnetospheres. The separation of the two pulsars in their orbits (Fig. 4 top) is typically $\sim 900,000 \mathrm{~km}$ or $3 \mathrm{lt}-\mathrm{s}$ (the distance light travels in three seconds). The large orbital inclination means that, at conjunction, the line-of-sight to one pulsar passes within about 0.15 lt-s of the other (Fig. 4 bottom). This is substantially smaller than the 0.45 lt-s radius of B's light cylinder, the point at which the co-rotation speed equals the speed of light, although much greater than the 0.004 lt-s light cylinder radius of A. As the pulsars move in their orbits, the line-of-sight from A passes through, and sweeps across, the magnetosphere of B, providing the opportunity to probe its physical conditions. The determination of changes in the radio transmission properties, including the dispersion and rotation measures, will potentially allow the plasma density and magnetic field structure to be probed. Additionally, the $\sim 70$-yr period geodetic precession will cause the line-of-sight to sample different trajectories through the magnetosphere of B. To a lesser extent, the $21-\mathrm{yr}$ orbital precession will also cause changes in the trajectory.

Close inspection of the flux density of the pulses from A (Fig. 5) reveals that a short occultation occurs, centered upon its superior conjunction (when the line-of-sight passes only 0.15 lt-s 
from B). The duration of the occultation is about 20-30 sec. Because the relative transverse velocity is about $660 \mathrm{~km} / \mathrm{s}$, the eclipsing region has a lateral extent of $\sim 15,000 \mathrm{~km}$ or $\sim 0.05 \mathrm{lt}-\mathrm{s}$, about $10 \%$ of the light cylinder radius of B. As far as we can tell at present, the eclipse occurs in every orbit and its extent is essentially the same at $680 \mathrm{MHz}$ and $1390 \mathrm{MHz}$, limiting the possible interpretations. One clue may come from the fact that the rate of spin-down energy loss from $\mathrm{A}$ is $\sim 3600$ times greater than that from B. In fact, at the light cylinder radius of B, the energy density of the relativistic wind from A is about two orders of magnitude greater than that from B, ensuring that the wind from A will penetrate deep into B's magnetosphere. We find that the energy densities due to the spin-down luminosity (assumed isotropic) emitted by A and the magnetic field of B are in balance at a distance $\sim 0.2$ lt-s from B, about $40 \%$ of the light cylinder radius of $\mathrm{B}$, assuming that the magnetic field can be calculated using the standard dipole formula (30). Within this distance, the energy field of B will dominate, while A will dominate outside. Even though this picture is roughly consistent with the lateral extent of the observed eclipse of A, it is likely that the strong departure of B's magnetosphere from an ideal case makes such calculations uncertain.

Because the point of pressure-balance is deep within the magnetosphere of $\mathrm{B}$, the actual penetration of the wind from A into B's magnetosphere will be a function of the orientation of the rotation and/or magnetic axes of B relative to the direction of the wind and hence will depend on the precessional and orbital phases of B. This is the most likely explanation for the large flux density and pulse-shape changes of B which are seen to vary with orbital phase (Fig. 1). We note that these changes are essentially the same between $680 \mathrm{MHz}$ and $3030 \mathrm{MHz}$. It seems most likely to us that such a broadband modulation arises from the impact of energy in the form of particles, gamma-rays or $44-\mathrm{Hz}$ electromagnetic radiation from the millisecond pulsar A upon the magnetosphere of B. We note that there is a strong indication of significant unpulsed radio emission from the system: the time-averaged pulsed flux density of the pulsars 
is about $1.8 \mathrm{mJy}$ at $1390 \mathrm{MHz}$ (Table 1), compared with a total flux density at this frequency of $7 \mathrm{mJy}(3)$. The $\sim 5 \mathrm{mJy}$ unpulsed emission probably arises in the impact region described above. We find it remarkable, with much of the magnetosphere of B blown away by the wind of A, that B still works as a pulsar. This suggests that the radio emission is probably generated close to the neutron star, providing a direct constraint on the emission height.

Conclusion. We have detected the binary companion of the millisecond pulsar J0737-3039 as a pulsar, making this the first known double-pulsar system. This discovery confirms the neutron star nature of the companions to recycled pulsars in eccentric binary systems and validates the suggested evolutionary sequences in which a companion star, having spun up the pulsar, forms a young pulsar in a supernova explosion (31). The highly-relativistic nature of this compact system opens up opportunities for much more stringent tests of relativistic gravitation than have been possible previously. Not only have we already measured four quantities attributable to, and consistent with, general relativity, but the mass-ratio $R$ is a new high-precision constraint that is independent of gravitational theories. Within a year or so we expect to measure the orbital decay due to emission of gravitational radiation. If the intrinsic value due to gravitational-wave damping can be extracted, it will allow tests of radiative aspects of gravitational theories mixed with strong-field effects. On somewhat longer time-scales of a few years we expect to detect several other relativistic effects such as geodetic precession of the pulsars' spin axes, spin-orbit coupling and other deviations, making this a superb testbed for relativity.

The detection of the companion as a pulsar also opens up the possibility of using each pulsar to probe the magnetosphere of the other. The energy flux from the millisecond pulsar is strongly affecting the pulse emission process in the companion and eclipses of the millisecond pulsar by the companion are also seen. Future measurements of orbital variations in pulse shapes, amplitudes, polarization and timing over a range of radio frequencies will give fascinating insights 
into magnetospheric processes in pulsars.

\section{References and Notes}

1. J. H. Taylor, Philos. Trans. Roy. Soc. London A 341, 117 (1992).

2. R. A. Hulse, J. H. Taylor, ApJ 195, L51 (1975).

3. M. Burgay, et al., Nature 426, 531 (2003).

4. V. Kalogera, et al., ApJ (2004). In press (astro-ph/0312101).

5. Receiver bandwidths used were $64 \mathrm{MHz}$ at $680 \mathrm{MHz}, 256 \mathrm{MHz}$ at $1390 \mathrm{MHz}$ and $576 \mathrm{MHz}$ at $3030 \mathrm{MHz}$.

6. http://pulsar.princeton.edu/tempo/.

7. T. Damour, J. H. Taylor, Phys. Rev. D 45, 1840 (1992).

8. J. H. Taylor, J. M. Weisberg, ApJ 345, 434 (1989).

9. I. H. Stairs, S. E. Thorsett, J. H. Taylor, A. Wolszczan, ApJ 581, 501 (2002).

10. M. Bailes, S. M. Ord, H. S. Knight, A. W. Hotan, ApJ 595, L49 (2003).

11. T. Damour, N. Deruelle, Ann. Inst. H. Poincaré (Physique Théorique) 44, 263 (1986).

12. T. Damour, J. H. Taylor, ApJ 366, 501 (1991).

13. T. Damour, N. Deruelle, Ann. Inst. H. Poincaré (Physique Théorique) 43, 107 (1985).

14. This is correct up to so-called first post-Newtonian $\left((v / c)^{2}\right)$ order $(7)$ and any intersection of the PK-parameter lines must be located on the $R$-line which will only deviate from that shown in Fig. 3 by corrections of order $(v / c)^{4}$. 
15. This is correct due to the usage of the DD timing model in our analysis and its definition of mass (13, 11).

16. T. Damour, R. Ruffini, C. R. Acad. Sc. Paris, Serie A 279, 971 (1974).

17. B. M. Barker, R. F. O’Connell, ApJ 199, L25 (1975).

18. J. M. Weisberg, R. W. Romani, J. H. Taylor, ApJ 347, 1030 (1989).

19. J. M. Weisberg, J. H. Taylor, ApJ 576, 942 (2002).

20. M. Kramer, ApJ 509, 856 (1998).

21. T. Damour, G. Schäfer, Nuovo Cim. 101, 127 (1988).

22. N. Wex, Class. Quantum Grav. 12, 983 (1995).

23. D. Bhattacharya, E. P. J. van den Heuvel, Phys. Rep. 203, 1 (1991).

24. G. S. Bisnovatyi-Kogan, B. V. Komberg, Sov. Astron. 18, 217 (1974).

25. N. Shibazaki, T. Murakami, J. Shaham, K. Nomoto, Nature 342, 656 (1989).

26. Z. Arzoumanian, J. M. Cordes, I. Wasserman, ApJ 520, 696 (1999).

27. E. P. J. van den Heuvel, C. de Loore, $A \& A$ 25, 387 (1973).

28. B. P. Flannery, E. P. J. van den Heuvel, $A \& A$ 39, 61 (1975).

29. S. E. Thorsett, D. Chakrabarty, ApJ 512, 288 (1999).

30. R. N. Manchester, J. H. Taylor, Pulsars (Freeman, San Francisco, 1977).

31. G. Srinivasan, E. P. J. van den Heuvel, $A \& A$ 108, 143 (1982). 
32. J. H. Taylor, J. M. Cordes, ApJ 411, 674 (1993).

33. We would like to thank John Sarkissian and other members of the Parkes multibeam team for their kind help with making the observations described in this paper. Extensive use was made of the PSRCHIVE pulsar analysis system developed by Aidan Hotan and colleagues (see http://astronomy.swin.edu.au/pulsar). We are grateful to Norbert Wex and Gerhard Schäfer for useful discussions. The Parkes radio telescope is part of the Australia Telescope which is funded by the Commonwealth of Australia for operation as a National Facility managed by CSIRO. MB, AP and ND'A acknowledge financial support from the Italian Ministry of University and Research (MIUR) under the national program Cofin 2001. FC is supported by NSF, NASA, and NRAO. DRL is a University Research Fellow funded by the Royal Society. 
Table 1: Observed and derived parameters of PSRs J0737-3039A and B using the DD timing model (13, 11). Standard $(1 \sigma)$ errors are given in parentheses after the values and are in units of the least significant digit(s). The parameters $A, B$ and $\delta_{r}$ in the DD model were assumed to be zero in the analysis. The distance is estimated from the dispersion measure and a model for the interstellar free electron distribution (32).

\begin{tabular}{|c|c|c|}
\hline Pulsar & PSR J0737-3039A & PSR J0737-3039B \\
\hline Pulse period $P(\mathrm{~ms})$ & $22.69937855615(6)$ & $2773.4607474(4)$ \\
\hline Period derivative $\dot{P}$ & $1.74(5) \times 10^{-18}$ & $0.88(13) \times 10^{-15}$ \\
\hline Epoch of period (MJD) & 52870.0 & 52870.0 \\
\hline Right ascension (J2000) & $07^{\mathrm{h}} 37^{\mathrm{m}} 51^{\mathrm{s}} .247(2)$ & - \\
\hline Declination (J2000) & $-30^{\circ} 39^{\prime} 40^{\prime \prime} .74(3)$ & - \\
\hline Dispersion measure DM $\left(\mathrm{cm}^{-3} \mathrm{pc}\right)$ & $48.914(2)$ & $48.7(2)$ \\
\hline Orbital period $P_{\mathrm{b}}$ (day) & $0.102251563(1)$ & - \\
\hline Eccentricity $e$ & $0.087779(5)$ & - \\
\hline Epoch of periastron $T_{0}(\mathrm{MJD})$ & $52870.0120589(6)$ & - \\
\hline Longitude of periastron $\omega(\mathrm{deg})$ & $73.805(3)$ & $73.805+180.0$ \\
\hline Projected semi-major axis $x=a \sin i / c(\mathrm{sec})$ & $1.41504(2)$ & $1.513(4)$ \\
\hline Advance of periastron $\dot{\omega}(\mathrm{deg} / \mathrm{yr})$ & $16.90(1)$ & - \\
\hline Gravitational redshift parameter $\gamma(\mathrm{ms})$ & $0.38(5)$ & \\
\hline Shapiro delay parameter $s$ & $0.9995(-32,+4)$ & \\
\hline Shapiro delay parameter $r(\mu \mathrm{s})$ & $5.6(-12,+18)$ & \\
\hline RMS timing residual $(\mu \mathrm{s})$ & 27 & 2660 \\
\hline Flux density at $1390 \mathrm{MHz}(\mathrm{mJy})$ & $1.6(3)$ & $0-1.3(3)$ \\
\hline Characteristic age $\tau$ (My) & 210 & 50 \\
\hline Surface magnetic field strength $B$ (Gauss) & $6.3 \times 10^{9}$ & $1.6 \times 10^{12}$ \\
\hline Spin-down luminosity $\dot{E}$ (erg/s) & $5800 \times 10^{30}$ & $1.6 \times 10^{30}$ \\
\hline Mass function $\left(\mathrm{M}_{\odot}\right)$ & $0.29097(1)$ & $0.356(3)$ \\
\hline Distance $(\mathrm{kpc})$ & \multicolumn{2}{|c|}{$\sim 0.6$} \\
\hline Total system mass $m_{\mathrm{A}}+m_{\mathrm{B}}\left(\mathrm{M}_{\odot}\right)$ & \multicolumn{2}{|c|}{$2.588(3)$} \\
\hline Mass ratio $R \equiv m_{\mathrm{A}} / m_{\mathrm{B}}$ & \multicolumn{2}{|c|}{$1.069(6)$} \\
\hline Orbital inclination from Shapiro $s$ (deg) & \multicolumn{2}{|c|}{$87(3)$} \\
\hline Orbital inclination from $(R, \dot{\omega})(\mathrm{deg})$ & \multicolumn{2}{|c|}{$87.7(-29,+17)$} \\
\hline Stellar mass from $(R, \dot{\omega})\left(\mathbf{M}_{\odot}\right)$ & $1.337(5)$ & $1.250(5)$ \\
\hline
\end{tabular}


Fig. 1. The intensity of radiation of $B$ as a function of orbital longitude relative to the ascending node of its orbit. Each panel shows a grey-plot of intensity over a phase range of 0.1 of pulsar rotational period, centered on the pulsed emission. Panels (A), (B) and (C) present observations at $680 \mathrm{MHz}, 1390 \mathrm{MHz}$ and $3030 \mathrm{MHz}$ respectively (summed over 6 orbits at $1390 \mathrm{MHz}$ and 2 orbits at each of the other frequencies), showing the similarity of the intensity variation over the wide frequency range and the changing pulse-shape with orbital longitude. Longitude in this diagram (and in Figs. 2 and 5) is strictly the sum of the longitude of periastron and the true anomaly. The vertical line at longitude $270^{\circ}$ represents the longitude of inferior conjunction of $\mathrm{B}$, when the two stars are at their closest on the sky and B is the nearer to the Earth.

Fig. 2. The effect of the Shapiro delay caused by the gravitational potential of B seen in the timing residuals of A. Residuals were averaged into 75 equal bins of orbital longitude. Top: timing residuals obtained by subtracting the model defined in Table 1 from the observed TOAs. Bottom: as top display, but with the Shapiro delay parameters $r$ and $s$ set to zero.

Fig. 3. The observational constraints upon the masses $m_{\mathrm{A}}$ and $m_{\mathrm{B}}$. The colored regions are those which are excluded by the Keplerian mass functions of the two pulsars. Further constraints are shown as pairs of lines enclosing permitted regions as predicted by general relativity (see text for details): (a) the measurement of the advance of periastron $\dot{\omega}$, giving the total mass $m_{\mathrm{A}}+m_{\mathrm{B}}=2.588 \pm 0.003 \mathrm{M}_{\odot}\left(\right.$ dashed line); (b) the measurement of $R \equiv m_{\mathrm{A}} / m_{\mathrm{B}}=x_{\mathrm{B}} / x_{\mathrm{A}}=$ $1.069 \pm 0.006$ (solid line); (c) the measurement of the gravitational redshift/time dilation parameter $\gamma$ (dot-dash line); (d) the measurement of Shapiro parameter $r$ giving $m_{\mathrm{B}}=1.2 \pm 0.3 \mathrm{M}_{\odot}$ (dot-dot-dot-dash line) and (e) Shapiro parameter $s$ (dotted line). Inset is an enlarged view of the small square which encompasses the intersection of the three tightest constraints, with the scales increased by a factor of 16 . The permitted regions are those between the pairs of parallel lines and we see that an area exists which is compatible with all constraints, delineated by the solid blue region. 
Fig. 4. The physical configuration of the binary system, at conjunction, as on 19 August 2003 (MJD 52870), showing the relative sizes of the two orbits and B's magnetosphere. At conjunction, the two neutron stars are separated by $\sim 2.8$ lt-s or about $800,000 \mathrm{~km}$. Top: view from above the orbital plane with the Earth to the right. The shaded segments indicate orbital phases where B is detected strongly (see Fig. 1). Also shown is the apsidal line, which is the major axis of the orbits, and the line-of-nodes, which is the intersection of the orbital plane and the plane through the center of mass of the system which is normal to the line-of-sight. Bottom: view from the side, showing the passage of the line-of-sight from A to the Earth through the magnetosphere of $\mathrm{B}$. The approximate position of the pressure balance between the relativistic wind from $\mathrm{A}$ and the magnetic field of $\mathrm{B}$ is indicated.

Fig. 5. The variation in flux density of A (in arbitrary units) at $680 \mathrm{MHz}$ and $1390 \mathrm{MHz}$, around superior conjunction (i.e. longitude $90^{\circ}$ ). The data are presented with 5 -sec time resolution and show the eclipse of the pulsar by the magnetosphere of B. 
Figure 1:

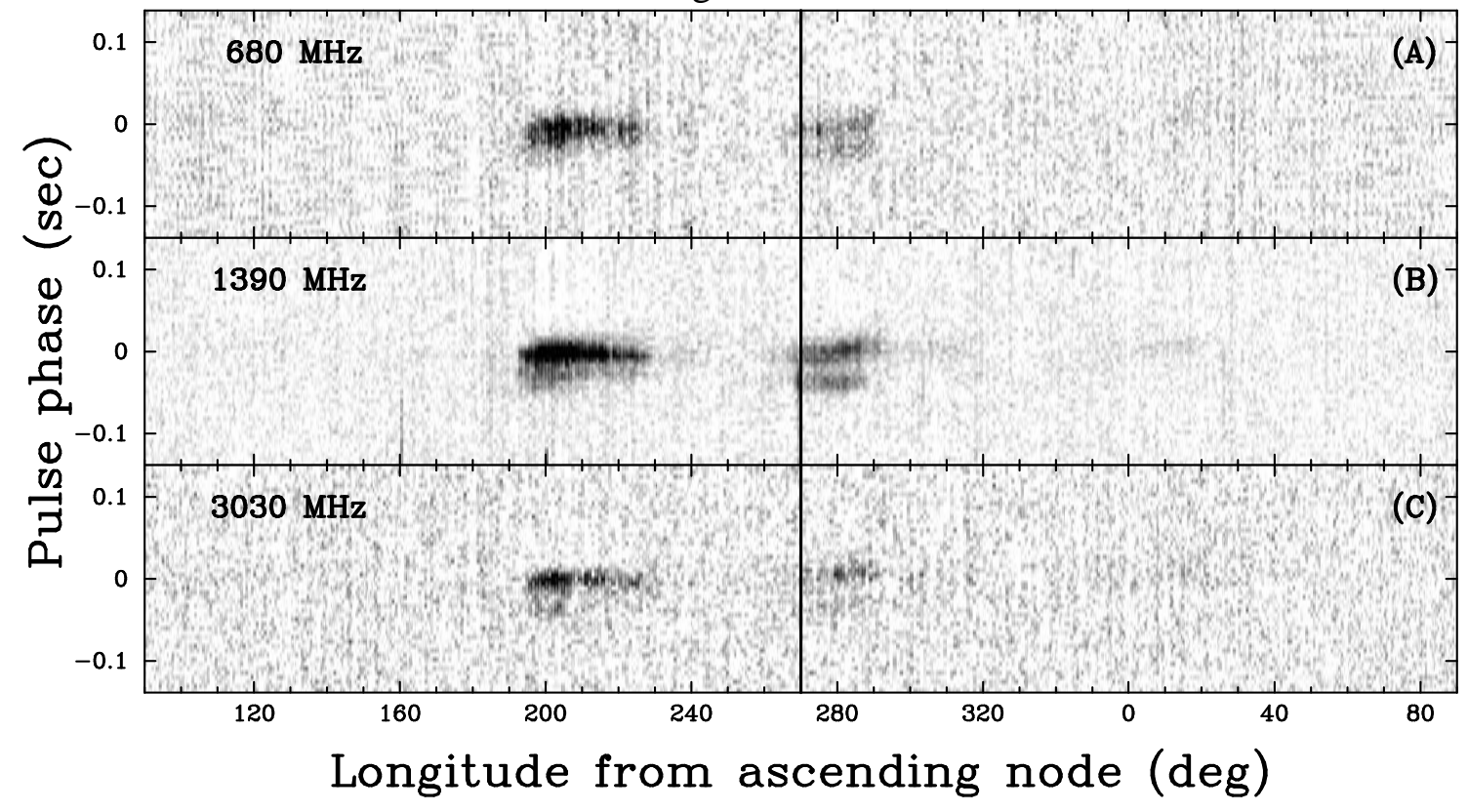


Figure 2:

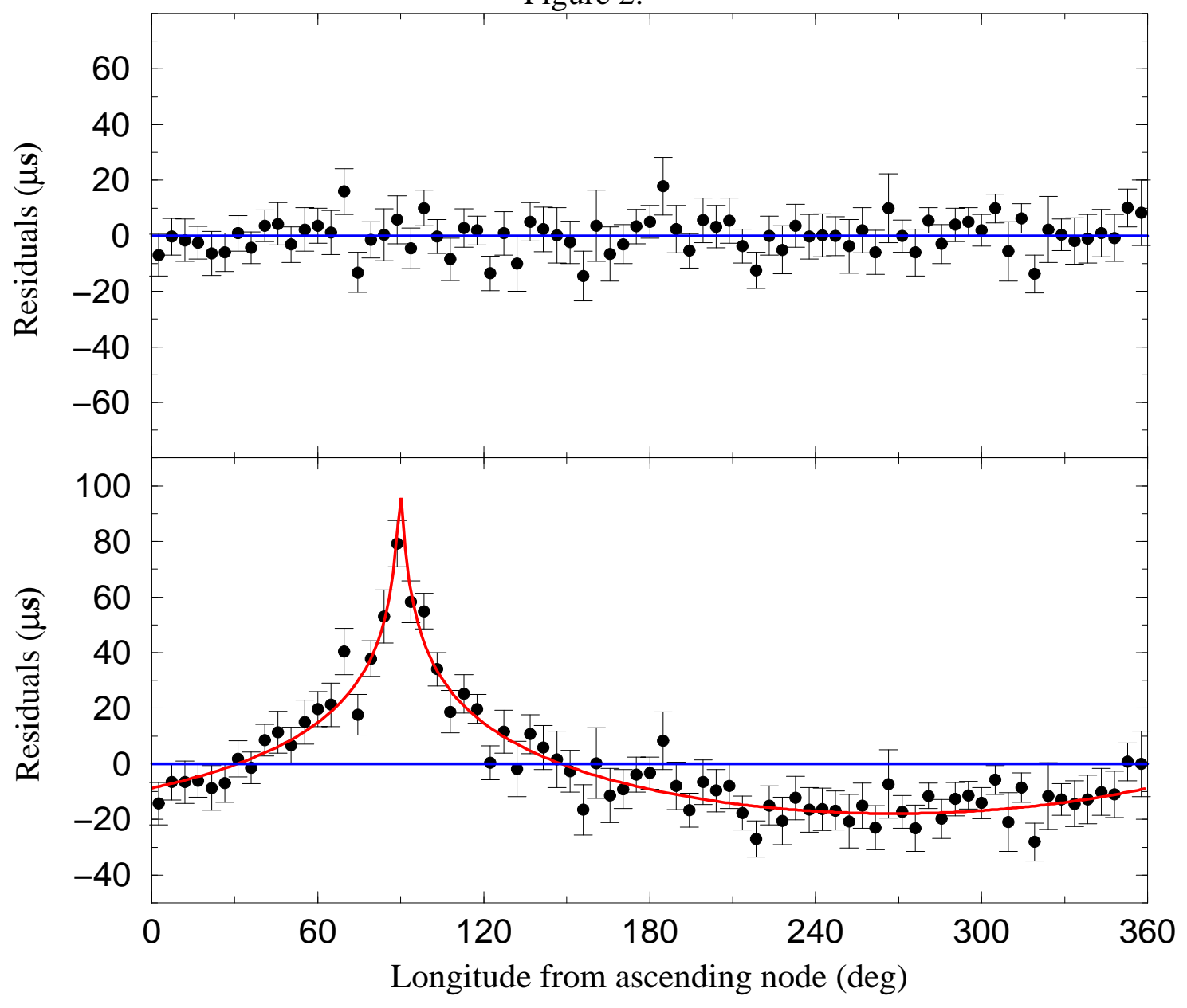


Figure 3:

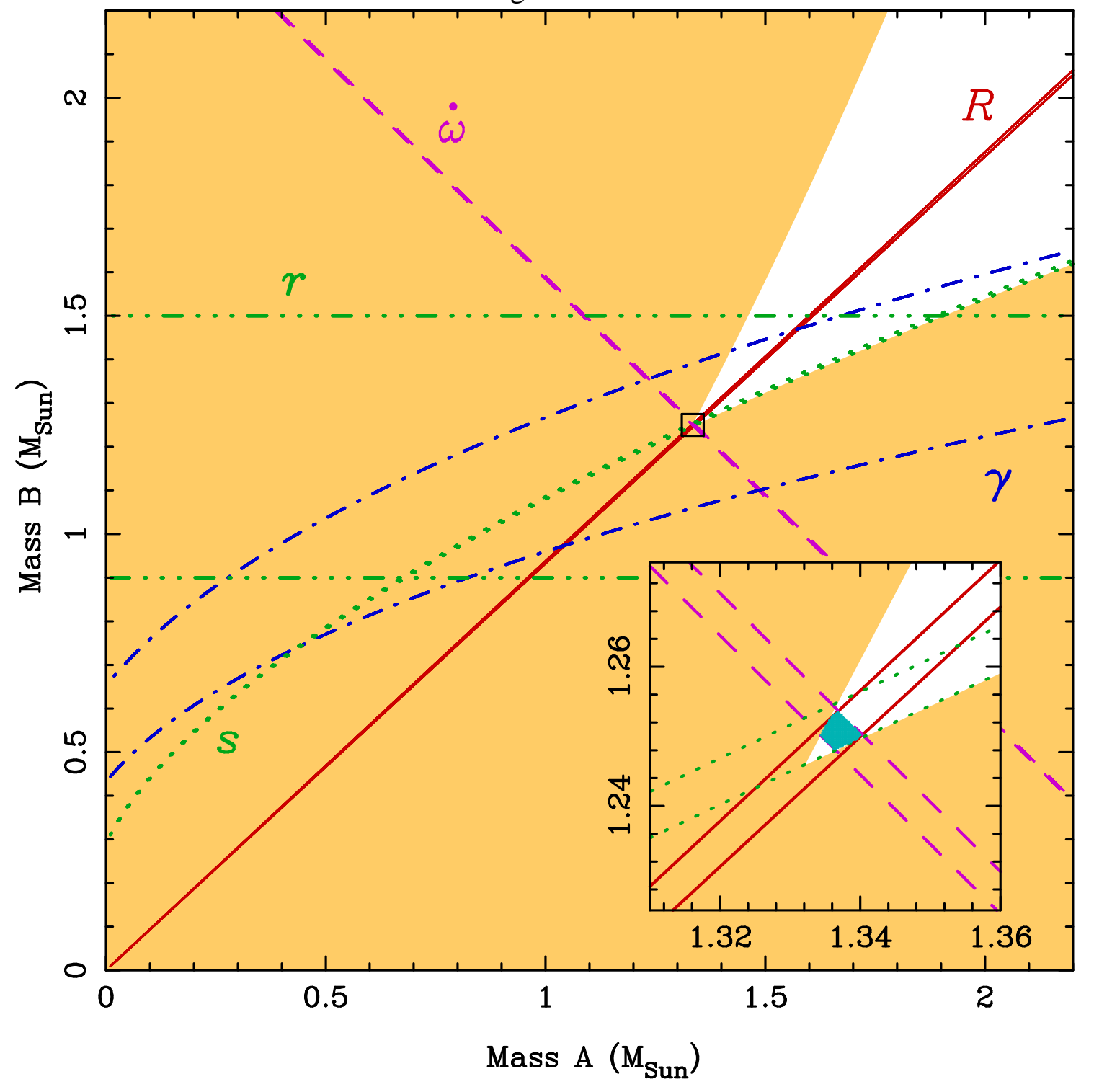


Figure 4:
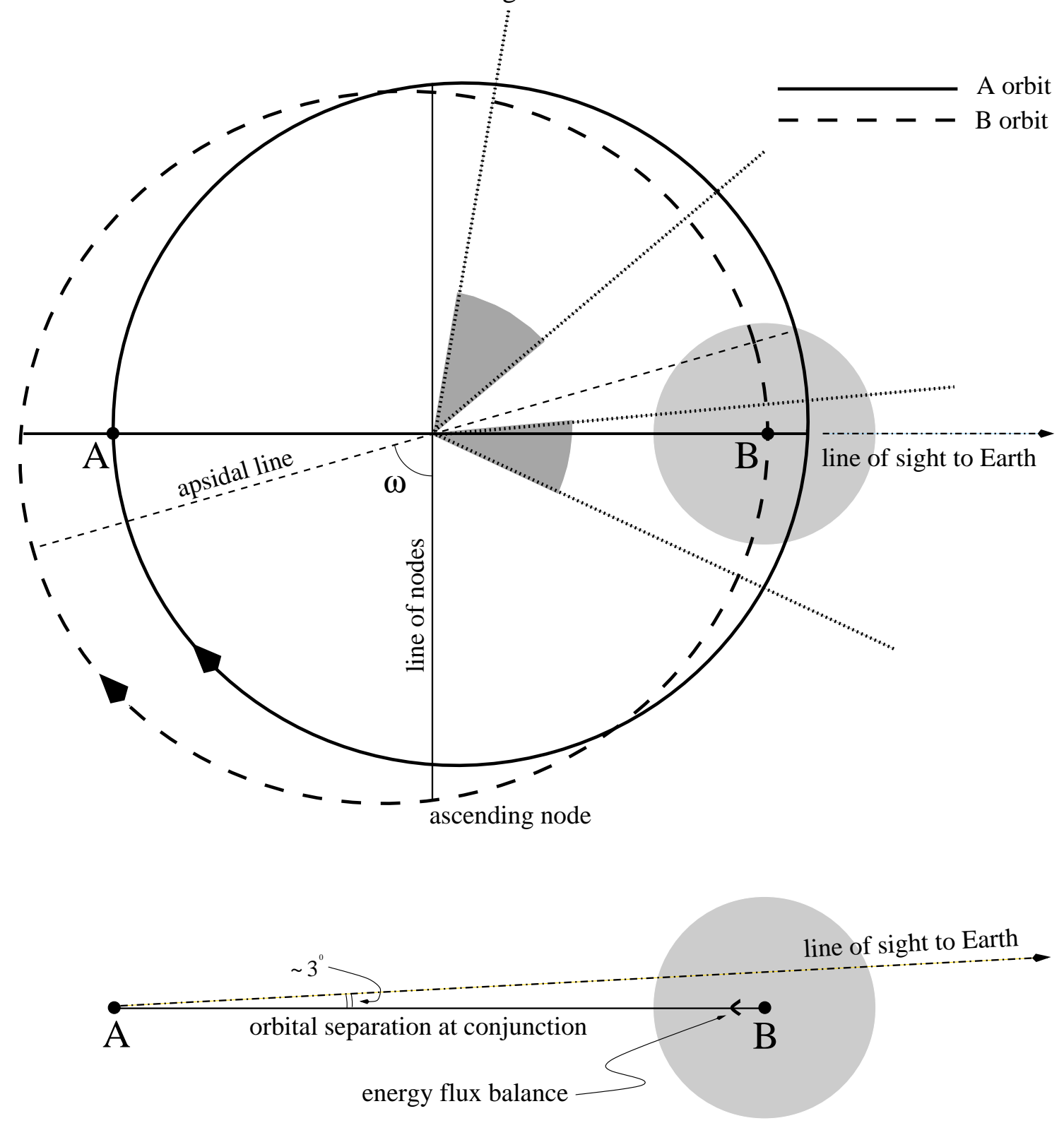
Figure 5:

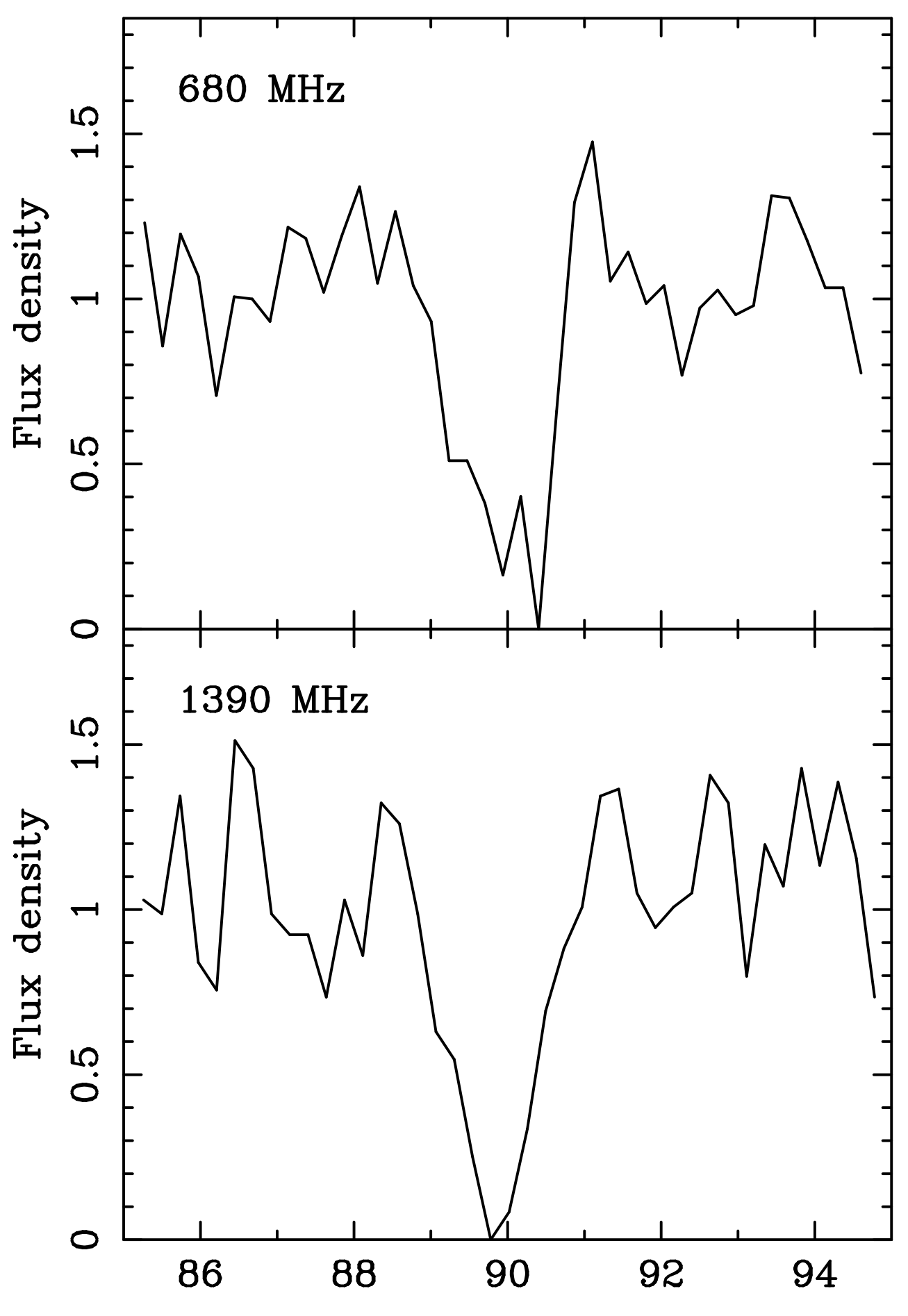

Longitude from ascending node (deg) 